\section{Fecal impaction causing pelvic venous compression and edema}

\author{
Sara Naramore, ${ }^{1}$ Faisal Aziz, ${ }^{2}$ Chandran \\ Paul Alexander, 3 Sosamma Methratta,4 \\ Robert Cilley, ${ }^{5}$ Dorothy Rocourt 5 \\ 1Department of Pediatrics, 2Department \\ of Vascular Surgery, ${ }^{3}$ Division of Pediatric \\ Gastroenterology, ${ }^{4}$ Department of \\ Radiology, 5Department of Surgery, Penn \\ State Milton S Hershey Medical Center, \\ PA, USA
}

\section{Abstract}

Chronic constipation is a common condition which may result in fecal impaction. A 13-yearold male with chronic constipation and encopresis presented with fecal impaction for three weeks. The impaction caused abdominal pain, distension, encopresis, and decreased oral intake. He was found in severe distress with non-pitting edema of his feet and ankles along with perineal edema. The pedal edema worsened after receiving a fluid bolus, so concern arose for venous compression or a thrombus. A Duplex Ultrasound demonstrated changes in the venous waveforms of the bilateral external iliac and common femoral veins without thrombosis. Manual disimpaction and polyethylene glycol 3350 with electrolytes resolved the pedal and perineal edema. Four months later, he had soft bowel movements without recurrence of the edema. A repeat Duplex Ultrasound was normal. We present a child in whom severe fecal impaction caused pelvic venous compression resulting in bilateral pedal and perineal edema.

\section{Introduction}

Childhood constipation is a common complaint leading to about $3 \%$ of visits to a general pediatrician and about $25 \%$ of visits to a pediatric gastroenterologist. 1 The worldwide prevalence of functional constipation in children varies between $0.7-29.6 \%$ and costs the United States alone approximately 3.9 billion Dollars per year in health care costs. In over $90 \%$ of patients with constipation, the diagnosis of functional constipation is given as no organic etiology is found. Full resolution is difficult, and follow-up is necessary as $50 \%$ of children will continue to have difficulty with constipation five years after treatment. Fecal impaction occurs in approximately a third of these children and needs to be managed promptly before serious complications occur. ${ }^{2}$ We report a unique case in which a child with severe fecal impaction developed bilateral lower limb edema with erythema due to pelvic venous compression. The edema resolved after disimpaction and a thorough bowel cleanout.

\section{Case Report}

A thirteen-year-old Caucasian male presented to the Penn State Hershey Emergency Department (PA, USA) with severe fecal impaction for three weeks. He had an episode of encopresis at school while taking $8.5 \mathrm{~g}$ of polyethylene glycol 3350 per day infrequently his pediatrician had prescribed $17 \mathrm{~g}$ by mouth once daily on a regular basis, but the encopresis experienced on this dose caused significant embarrassment to the patient resulting in noncompliance. Two days prior to admission, he developed severe abdominal pain with cramping, vomiting, decreased oral intake, and diminished urine output. He also complained of pallor, diaphoresis, insomnia, and restlessness. He was treated with magnesium citrate 10 ounces orally and a saline enema at an outside hospital without relief and eventual worsening of his symptoms. He had a significant history of chronic functional constipation beginning at the age of two years consisting of large, hard, painful stools that led to a pattern of withholding for upwards of eight days. He struggled to become toilet trained and was seen in our Pediatric Gastroenterology clinic for a first visit at three years of age. He had passed meconium shortly after birth, and his medical history only included a repair of a penile hypospadias. His diet contained less than sixteen ounces of milk per day, and he was growing well with weight and height in the $85^{\text {th }}$ percentile and $50^{\text {th }}$ percentile, respectively. A rectal examination demonstrated normal sphincter tone and a posterior shelf. Daily oral mineral oil was prescribed; however, he was lost to follow-up thereafter. He was seen by his pediatrician for annual health visits since 10 years of age and concerns of constipation with encopresis were addressed appropriately - including regular daily osmotic laxative therapy, letters to school teachers to facilitate the patient's use of the restroom as needed, and high fiber diet. His parents reportedly attempted to manage his constipation with intermittent doses of oral mineral oil, senna, naturopathic therapy, and glycerin or bisacodyl suppositories as needed primarily to manage acute distress as they were wary of long term dependence on medications. With increasing age, the patient was given more responsibility for adhering to his therapy. To his parents, he appeared to be in control of his constipation for the most part, till onset of the current illness. His diet was predominantly low fiber
Correspondence: Chandran Paul Alexander, Division of Pediatric Gastroenterology, Hepatology and Nutrition, Department of Pediatrics, Penn State Milton S Hershey Medical Center, Mail Code H085, 500 University Drive, Hershey, PA 17033, USA.

Tel.: +1.717.531.1043 - Fax: +1.717.531.0653.

E-mail: calexander@hmc.psu.edu

Key words: Pedal edema; duplex ultrasound; polyethylene glycol 3350 with electrolytes; constipation; fecal impaction.

Contributions: SN reviewed the existing literature, drafted the initial manuscript, approved the final manuscript; FA reported the findings on the Doppler Ultrasound, reviewed and revised the manuscript, approved the final manuscript; CPA supervised the patient care, reviewed and revised the manuscript, approved the final manuscript; SM, reported the findings on the abdominal radiograph, reviewed and revised the manuscript, approved the final manuscript; $\mathrm{RC}$ supervised the fecal disimpaction procedure, reviewed and revised the manuscript, and approved the final manuscript; DR assisted with the fecal disimpaction, reviewed and revised the manuscript, and approved the final manuscript.

Conflict of interest: the authors declare no conflict of interest.

Received for publication: 8 May 2015.

Revision received: 17 Juy 2015.

Accepted for publication: 21 July 2015 .

This work is licensed under a Creative Commons Attribution NonCommercial 3.0 License (CC BYNC 3.0).

(C) Copyright S. Naramore et al., 2015

Licensee PAGEPress, Italy

Pediatric Reports 2015; 7:5999

doi:10.4081/pr.2015.5999

with very limited intake of vegetables or fruits.

Upon arrival to our hospital, he was in constant, severe discomfort and restless, but preferentially lying on his sides. His temperature was $36.4^{\circ} \mathrm{C}$, heart rate 116 beats per minute, and blood pressure $126 / 93 \mathrm{mmHg}$. His weight was $68 \mathrm{~kg}$ (93rd percentile) and height was 168 $\mathrm{cm}$ ( $76^{\text {th }}$ percentile). He had dry mucosal membranes and skin. His abdomen was moderately distended, firm to palpation with diffuse tenderness, and had palpable hard fecal masses in the suprapubic area and bilateral lower quadrants. He had mild bilateral non-pitting pedal edema. Perianal inspection revealed oozing, liquid brown stool with no skin tags. A digital rectal examination revealed a large, hard ball of stool impacted in the distal rectum. Inguinal lymphadenopathy and scrotal edema were absent. An abdominal radiograph demonstrated a large stool burden causing rectal dilation 
of $10 \mathrm{~cm}$ with distention of the sigmoid colon extending upwards to the level of the umbilicus (Figure 1). Laboratory testing revealed the following results which were all within normal limits: sodium $140 \mathrm{mmol} / \mathrm{L}$, potassium 4.1 $\mathrm{mmol} / \mathrm{L}$, chloride $100 \mathrm{mmol} / \mathrm{L}$, bicarbonate 24 $\mathrm{mmol} / \mathrm{L}$, blood urea nitrogen $11 \mathrm{mg} / \mathrm{dL}$, creatinine $0.61 \mathrm{mg} / \mathrm{dL}$, and glucose $91 \mathrm{mg} / \mathrm{dL}$. His complete blood cell count, thyroid function studies, tissue transglutaminase IgA and total IgA were normal. He was given a parenteral normal saline bolus for rehydration. Within thirty minutes of the fluid bolus, he developed worsening pedal edema and overlying erythema spreading upwards from his feet to his knees. Due to a concern for deep venous thrombosis, a Duplex Ultrasound was performed; it did not reveal a thrombus, but showed changes in the waveforms in his common femoral and external iliac veins bilaterally, indicating pelvic venous compression (Figure 2A,B). D-dimer and coagulation studies were obtained and were within normal limits. The pediatric surgeons performed a manual disimpaction under general anesthesia due to his worsening clinical status. A three-digit rectal examination encountered perineal edema and a large fecal impaction, which was manually removed with abdominal compression and transanal disruption. Nine liters of crystalloid solution were given in serial irrigations and resulted in multiple stool masses being removed. Over the next three days, his bowel was sufficiently cleaned out with sixteen liters of polyethylene glycol 3350 with electrolytes infused via a nasogastric tube. A repeat abdominal radiograph confirmed a significant reduction in the stool burden and decompression of his entire colon. His pedal and perineal edema resolved, and he was discharged on maintenance polyethylene glycol

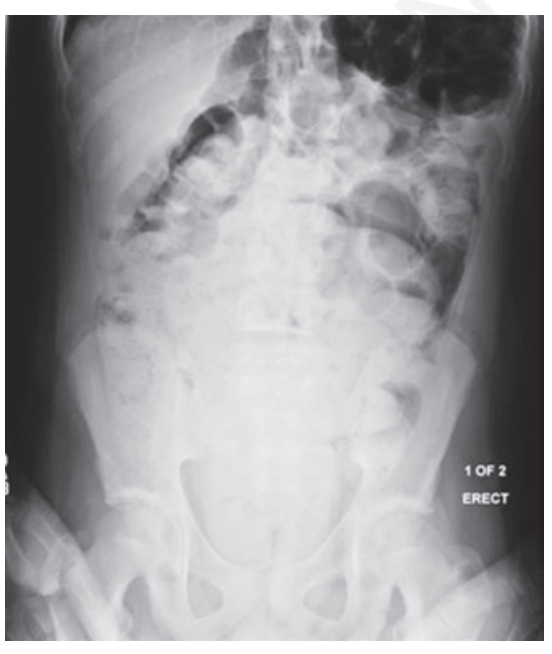

Figure 1. Abdominal radiograph showing fecal impaction.
3350 solution $17 \mathrm{gm}$ twice daily and Bisacodyl $10 \mathrm{mg}$ tablet once daily.

One week later he had a barium enema which showed a posterior rectal shelf along with a change in caliber of the distal rectum with spiculations. These findings created concern for decreased colonic motility. Four months after discharge, he continued to have soft daily bowel movements with compliance to maintenance laxative therapy. The pedal and perineal edema had resolved. He had a good appetite and denied abdominal discomfort. Serial clinical examinations at two follow up visits did not reveal recurrence of palpable mass per abdomen. An abdominal radiograph one month later demonstrated minimal residual stool, and a repeat Doppler ultrasound showed normal venous waveforms (Figure 2C,D). Hence, imaging studies for evaluating other causes of extrinsic venous compression were not pursued. Further investigation of the etiology of the constipation, including a rectal biopsy and anorectal and colonic manometry, were deferred at this time due to the resolution of the constipation. Subsequent annual visits to his pediatrician's office found him to be adhering to a higher fiber diet, daily laxative as prescribed, with no reported difficulty in stooling and steady growth. His weight had gone up to $70.5 \mathrm{~kg}$ at 14 years of age and to 81.4 $\mathrm{kg}$ at 15 years of age.
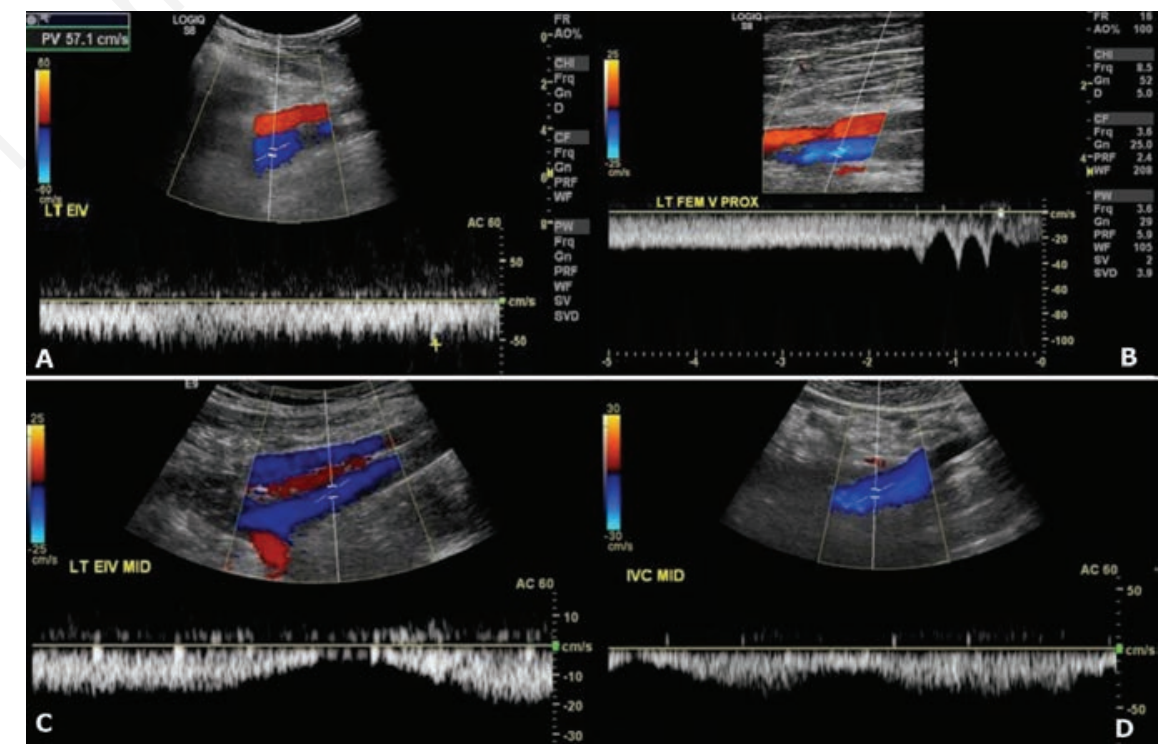

Figure 2. A) Color duplex showing evidence of flow inside the left external iliac artery (red) and left external iliac vein (blue). Absence of respiratory variations in venous waveforms suggests a proximal obstruction; B) Absence of respiratory variations in venous wave-form of the left femoral vein suggests a proximal obstruction. C) Color duplex after fecal disimpaction with normal venous waveforms in left external iliac vein and $D$ ) inferior vena cava. 
Children may also present with abdominal pain, vomiting, anorexia, and weight loss. ${ }^{3}$ These symptoms cause a significant amount of psychosocial stress, physical morbidity, and impaired quality of life for the child. ${ }^{2}$

Less commonly, fecal impaction secondary to chronic constipation has been associated with various serious clinical presentations, such as stercoral ulcers of the rectosigmoid with perforation, bleeding, peritonitis, and bacteremia. ${ }^{4}$ Other serious complications can include intussusception caused from an intramural fecolith, acquired megarectum and megacolon, rectovaginal fistula, appendicitis, and appendiceal colic.5,6 The large stool burden can cause compression of other organs resulting in sciatica, hydronephrosis, malfunction of a peritoneal dialysis catheter, urinary retention, and urinary tract infections. ${ }^{7-11}$

In our patient, the prolonged fecal impaction with massive colonic fecal loading led to increased intra-abdominal pressure causing pelvic deep venous compression with resultant bilateral pedal edema. The impedance of venous return caused an increase in capillary hydrostatic pressure in the lower extremities which forced fluid into the interstitial spaces. ${ }^{12}$ The venous stasis placed him at risk for a pelvic vein thrombosis and embolism. Hence, the intra-abdominal pressure needed to be relieved quickly in order to prevent further morbidity.

\section{Conclusions}

Therefore it is important to treat constipation in children appropriately before complications arise. In the majority of cases, it can be managed in an outpatient setting with oral laxatives, adequate hydration, and a balanced diet. Fecal impaction develops in about a third of children and requires aggressive treatment with oral laxatives and rectal therapy. ${ }^{2}$ Thus, physicians should provide timely management, education especially about initial worsening of encopresis with laxatives and of the need to persist with medical therapy till establishment of regular toileting habits and resolution of soiling episodes, psycho-social support and more frequent follow-up in order to ensure a good long-term outcome.

\section{References}

1. Pashankar DS. Childhood constipation: evaluation and management. Clin Colon Rectal Surg 2005;18:120-7.

2. Mugie SM, Di Lorenzo C, Benninga MA. Constipation in childhood. Nat Rev Gastroenterol Hepatol 2011;8:502-11.

3. Rajindrajith S, Devanarayana NM. Constipation in children: novel insight into epidemiology, pathophysiolocgy, and management. J Neurogastroenterol Motil 2011;17:35-47.
4. Halawi HM, Maasri KA, Mourad FH, Barada KA. Faecal impaction: in-hospital complications and their predictors in a retrospective study on 130 patients. Colorectal Dis 2012;14:231-6.

5. Buchta RM. Intussusception in a 14-yearold boy secondary to an intramural fecalith. Am J Dis Child 1978;132:1140-1.

6. Singh JP, Mariadason JG. Role of the faecolith in modern-day appendicitis. Ann R Coll Surg Engl 2013;95:48-51.

7. Djeddi D, Vanrenterghem A, Hamriti A. An unusual case of hydronephrosis in a child. Arch Pediatr 2011;18:193-4;215-6.

8. Frischhut B, Ogon M, Trobos S, Judmaier W. Sciatica as a manifestation of idiopathic megacolon: a previously undescribed causal relationship. J Pediatr 1998; 133:449.

9. Rowan-Legg A. Managing functional constipation in children. Paediatr Child Health 2011;16:661-5.

10. Stonehill WH, Smith DP, Noe HN. Radiographically documented fecal impaction causing peritoneal dialysis catheter malfunction. J Urol 1995;153:4456.

11. Ruoss KA, O'Sullivan R. Chronic constipation causing obstructive uropathy in an adolescent male. Pediatr Emerg Care 2008;24:462-3.

12. Jacobs MK, McCance KL, Stewart ML. Leg volume changes with EPIC and posturing in dependent pregnancy edema. Nurs Res 1986;35:86-9. 